\title{
A POWERFUL RANGE-DOPPLER CLUTTER REJECTION STRATEGY FOR NAVIGATIONAL RADARS
}

\author{
T.K. Bhattacharya and P.R.Mahapatra
}

Department of Aerospace Engineering Indian Institute of Science Bangalore-560012, INDIA

\section{ABSTRACT}

Navigational radars are used to detect and continuously track a number of aircraft. In strongly cluttered environment, this can lead to broken and interrupted tracks. This could be hazardous specially in the terminal areas and approach corridors. Hence the optimum rejection of clutter is a primary requirement in navigational radars. This paper describes a method to combat arbitrary delay-Doppler clutter. The problem is approached via the signal ambiguity function. Matched filter receiver is assumed and transmitted signal parameters are optimized to achieve the clutter rejection capability. The signal set chosen is the family of frequency coded constant amplitude pulse bursts, and a criterion function is derived in terms of the frequencies of individual subpulses which has been optimized for a variety of clutter distributions. The results obtained clearly bring out the effectiveness of the method.

\section{INTRODUCTION}

Navigational radars traditionally provide information regarding aircraft. In more modern applications involving large scale automation of the navigational and air traffic control functions, the radars are required to provide precise and continuous track information on aircraft in a multiple target environment. However, in strongly cluttered regions, the detection of the aircraft by the radar becomes uncertain, resulting in broken and interrupted tracks. In dense traffic and highly dynamic navigational situations such as terminal areas and approach corridors, any significant loss of track would be hazardous. Thus the need for agile and situation-specific rejection of 2dimensional (range and Doppler) clutter is extremely important for modern navigational radars. This paper presents a method which optimizes the detection performance of the radar for any given clutter distribution in the delayDoppler plane of the radar. The method is versatile and powerful enough to handle arbitrary distributions, and yet is computationally tractable even on minicomputers and lower end mainframes of the type available at typical navigational facilities.

The problem has been formulated using signals termed Frequency-Shift Keyed (FSK), compact pulse bursts. This family has certain highly desirable properties as described later. Using this signal family, specific results are derived and presented for specific interference distributions.

\section{BACKGROUND}

The interference to a radar return consists of a superposition of noise and clutter i.e. the combined returns received from unwanted scatterers. Most often the most severe problem is to detect the target(s) in presence of these scatterers; it may not be necessary to resolve the individual objects causing this interference. Also, most often, clutter returns are strong enough to "bury" the echo power received from the target(s).

In general, an individual scatterer would have both a range displacement and a radial velocity relative to the target of interest and thus can be represented as a strength weighted point on a delay-Doppler plane centered on the target delay and Doppler. The most general clutter distribution may be represented by a continuous distribution of such points in the delay-Doppler plane. We refer to it as 2dimensional clutter or delay-Doppler clutter.

It is well known that noise effects can be minimized through the use of a matched filter receiver. For reducing clutter effects, however, specific design of the radiated signal envelope and/or receiver filter characteristics is necessary. Ideally, the use of a mismatched filter and the simultaneous optimization of the signal-filter combination should lend more versatility for clutter rejection than an optimum signal-matched-filter pair. However, Rihaczek [1] has shown that a properly designed transmitted waveform, along with its matched filter, performs almost as well as an optimum signal-mismatched-filter pair in most realistic situations, barring some very special clutter 
distributions. On the other hand, matched filters are, in general, simple to realize and have the added advantage of being inherently optimum against the white-noise component of the interference. For these reasons, in this paper the matched filter assumption is made and a technique for the design of optimum transmitted waveforms is presented. For discrete coded signals the matched filter assumption reduces the parameters of optimization by a factor of 2 . Since computational efforts for multivariable optimization grows exponentially with the problem dimensionality, this reduction in the number of optimization parameters greatly improves the tractability of the optimization, especially for long pulse codes.

If no a priori information is available about the target scenario, all signals are equally good or bad since it is known that no signal can have good overall behaviour throughout the delay-Doppler plane. This conclusion follows from the constant volume property of the signal ambiguity function. The range-Doppler ambiguity function serves as a natural tool for signal design against 2-D clutter. The ambiguity function has been used frequently in the past to understand clutter rejection behaviour of transmitted signal-receiver filter combinations and to design clutter-resistant signals and/or filters.

One of the earliest attempts to design a filter to obtain optimum performance against clutter was by Urkowitz [2] who considered the clutter as the only form of interference. he showed that the filter which maximizes the Signal-to-Interference Ratio (SIR) for clutter which is stationary with respect to the target has a frequency response which is the inverse of the spectrum of the transmitted signal. Since the frequency spectrum of any physically realizable signal has to approach zero on both sides of the spectrum, the ideal clutter filter, as suggested by him, becomes unrealizable, since it will have to have infinite bandwidth. The drawbacks of the 'Inverse filter' are discussed by Turin [3].

The combined interference by noise and clutter (stationary) was first considered by Manasse [4]. He showed that when the clutter is uniformly distributed in range, the optimum clutter rejecting signal is an impulse of infinitesimal duration, leading to a signal of infinite bandwidth and peak-power. When any reasonable restriction is put on the signal bandwidth, he showed, that the optimum signal is one which has a flat spectrum over the band of interest.

Ares [5] made a physical approach to the general problem of independently optimizing the signal and the filter and considered the case of clutter extended in range, but confined in Doppler to a narrow strip parallel to the delay axis.
Delong and Hofstetter [6] have also studied the general problem but confining the signals and receiver impulse responses to uniformly spaced, phase- and magnitude-tapered, pulse trains. They have evaluated the performance of both matched and "clutter" filters for general waveforms and have devised an iterative technique for optimizing the clutter filter performance for pulse train signals. In a later work [7] the same authors have evolved an algorithm for designing signal waveforms under a dynamic range constraint i.e., with a limit on the ratio of the maximum to the minimum amplitude of the transmit signal. Their approach resulted in a nonlinear programming formulation for the optimization of SIR.

The design of an optimum mismatched filter response for detecting a given signal in clutter has been considered by Stutt and Spafford [8]. They have used the techniques of variational calculus to minimize the output clutter power subject to certain constraints on the signal and filter responses. They obtained a linear integral equation, the solution of which leads to the optimum receiver filter, and they have suggested a method for solving the equation. Spafford's work [9], has extended this theory and outlined an iterative procedure to obtain the optimum signal-filter pair.

Recently Ziomek and Sibul [10] have extended the approach in [6] to formulate the problem of optimal signal design for the detection of a doubly-spread target against general clutter/reverberation. The problem has been posed as a number of nonlinear optimization problems. The problem of point target detection against 2-D clutter would be a special case of their formulation. However, solutions to the optimization problem have not been attempted by them, and no results are available.

Since radar detection is more frequently clutter-limited rather than noise-limited, mitigation of clutter effects is a strong requirement in radar system design. When the clutter is one-dimensional, essentially spread along the range axis only ( e.g. ground clutter), the methods of combating its effect are fairly straightforward and usually consist of using a simple waveform with the receiver filter tuned in terms of its frequency characteristics. However, when the clutter is two-dimensional, distributed along the range and velocity (Doppler) dimensions, the clutter alleviation strategy becomes more complex. A potent method of combating 2-D clutter effects is to choose transmitted signal with a large time-bandwidth product (TB), the received signal being processed through a matched or a mismatched filter.

For specific clutter distributions, wellknown large TB signals, such as the chirp signal, have been used along with their matched filters. However, when the clutter distribution is arbitrary, such relatively 'simple' signals do 
not necessarily perform well in all cases. This follows from the limited number of control parameters for such signals, which restricts the ability of the waveform to adapt to the clutter distribution in the delay-Doppler plane. A more modern approach is to have discrete-coded signals which offer more control parameters for finer control of the ambiguity surface.

\section{FORMULATION}

Dense clutter may be described as a distribution $\mathrm{p}(\tau, v)$ over the delay-Doppler plane. A measure of the detectability of the target is the signal-to-interference ratio (SIR), which is the ratio between the target signal and the interference (clutter+noise), both observed at the delay and Doppler corresponding to those of the target.

The output of the receiver filter for a given transmitted signal is given by the crosscorrelation function

$$
\left|X_{12}(\tau, v)\right|^{2}=\left|\int_{\infty}^{\infty} s_{1}(t) s_{2}^{*}(t-\tau) e^{-j 2 \pi v t} d t\right|^{2}
$$

where $s_{1}(t)$ is the transmitted signal and $s_{2}(t)$ is the signal to which the receiver is matched. Under a matched filter assumption,

$$
s_{1}(t)=s_{2}(t)=s(t)
$$

and the output of the receiver is given by the ambiguity function of the transmitted signal

$$
|X(\tau, v)|^{2}=\left|\int_{\infty}^{\infty} s(t) s^{*}(t-\tau) e^{-j 2 \pi v t} d t\right|^{2}
$$

Without loss of generality, the origin of the $(\tau, v)$ plane may be located at the delay and Doppler corresponding to those of the target. Then the output $S$ of the receiver filter at $(0,0)$ due to the target is

$$
S(0,0)=|X(0,0)|^{2}=E_{S}^{2}
$$

Where $E_{S}$ is the energy content of the signal $s(t)$. Also, the output of the receiver due to clutter at the target delay and Doppler is given by the integral

$$
C(0,0)=\int_{\infty}^{\infty} p(\tau, v)|x(\tau, v)|^{2} d \tau d v
$$

If $\left|x_{0}\right|^{2}$ is the normalized ambiguity function, (i.e. ambiguity function for the signal normalized to unit energy), then a well-known property of the ambiguity function [11] is

$$
\left|X_{0}(\tau, v)\right|^{2}=E_{s}^{2}|X(\tau, v)|^{2}
$$

Hence from eq (4),

$$
C(0,0)=E_{s}^{2} \cdot C_{0}(0,0)
$$

Where, $C_{0}$ refers to the clutter output for a unit energy transmitted signal.

Also, if the noise spectral density (onesided) at the input of the receiver is $\mathrm{N}_{0}$, then the noise at the output of the receiver is given by

$$
N=N_{0} E_{s}
$$

Therefore, the SIR at the output of the receiver is, from eqs (3)-(5),

$$
\begin{aligned}
\text { SIR } & =\frac{S(0,0)}{N+C(0,0)}=\frac{E_{S}^{2}}{N_{0} \cdot E_{s}+C(0,0)} \\
& =\frac{1}{\frac{N_{0}}{E_{S}}+\frac{C(0,0)}{E_{S}^{2}}} \\
& =\frac{1}{N_{0} / E_{s}+C_{0}(0,0)}
\end{aligned}
$$

The denominator of eq (8) consists of two terms. The first term $\left(\mathrm{N}_{0} / \mathrm{E}_{\mathrm{s}}\right)$ depends on the energy of the signal and can be decreased, for a given receiver noise level, only by increasing the energy of the transmitted signal. The second term is the contribution due to the clutter and is independent of the energy of the transmitted signal. However, since the normalized ambiguity function is strongly dependent on the shape (i.e. complex envelope) of the transmitted signal, its weighted integral $C_{0}$ will also, in general, be dependent on the wave shape. Any reduction in this factor can therefore be brought about only by designing a suitable signal waveform to minimize $\mathrm{C}_{0}$.

An efficient optimization procedure would be to express the signal in parametric form and then optimize the parameters minimizing $C_{0}$ for a given clutter distribution $\mathrm{p}(\tau, v)$. To facilitate this procedure, signals of specified families may be chosen. In this paper we consider constantamplitude compact frequency coded pulse bursts and formulate the problem of optimization against arbitrary delay-Doppler clutter distributions.

Frequency coded rectangular compact pulse bursts form a frequently used class of signals in modern high performance radars because of its obvious advantages over other classes of coded bursts. An FSK burst of $N$ subpulses have a TB of $\mathrm{N}^{2}$ as compared to $\mathrm{N}$ for a PSK burst of same 
length. Also this offers large number of optimization parameters ( $N$ for an $\mathrm{N}$ pulse long burst) for better control of the ambiguity behavior.

Some limited efforts have been made by a number of other authors [12-15], to study the performance of FSK bursts for clutter rejection. The approach in most cases, however, has been to choose a certain coding structure and then study the performance of the code in presence of interference. Such an approach severely limits the adaptability of the resultant waveform to cater to different target scenarios. An effort has been made, in this paper, to formulate the performance criterion in terms of the FSK parameters ( namely the frequency of subpulses) and to optimize the performance with respect to the parameters of the signal.

As discussed previously, the problem of maximizing SIR reduces to the minimization of the normalized clutter integral, $C_{0}$, in eq (4). The evaluation of $\mathrm{C}_{0}$ for an FSK burst is highly computation intensive and to effectively use the formulation the evaluation of the clutter integral must be simplified. The piecewise invariance of the parameters of the FSK burst can be used to effect this simplification.

\section{OPTIMIZATION CRITERION}

The complex envelope of the constantamplitude frequency coded compact pulse bursts is described as

$$
s(t)=\sum_{i=1}^{N} g_{i}(t) e^{j 2 \pi f_{i} t}
$$

where $f_{i} \quad i=\{1, \ldots, N\}$ refers to the frequency of the $i^{t h}$ subpulse and $g_{i}(t)$ is the gate function given in terms of a unit step function $u(t)$ as

$$
g_{j}(t)=u\{t-(i-1) T\}-u\{t-i T\}
$$

Let

$$
\tau=(I+F) T
$$

i.e. I is the integer part of any shift in delay and $F$ the fractional part. Therefore,

$$
I=0,1,2, \ldots,(N-1) \text { and } 0<F<1
$$

Substituting the value of $s(t)$ as given by eq (9) and using eq (10) we get, after simplification of eq (2), the ambiguity function of the FSK burst as

$X(\tau, v)=\sum_{i=I+1}^{N} e^{j u\left[2(I+F) f_{i-I}+x_{i}(2 i-1+F)\right]} \frac{\operatorname{Sin}\left(u x_{i}(1-F)\right)}{x_{i}}$

$+\sum_{k=1+2}^{N} e^{j u\left[2(I+F) f_{\left.k-I-I^{+} y_{k}(2 k-2+F)\right]} \frac{\operatorname{Sin}\left(u y_{k} F\right)}{y_{k}}\right.}$

where

$$
\begin{aligned}
& u=\pi / N \\
& x_{i}=v+f_{i}+f_{i-I} \quad \text { and } \\
& y_{k}=v+f_{k}+f_{k-I-1}
\end{aligned}
$$

Eq (11) can be used to obtain a fairly tractable expression for the clutter integral $C$, which can be used as the performance criterion and can be evaluated for a given distribution of $p(\tau, v)$ and optimized w.r.t $f_{i}$ 's to obtain the optimum signal for that case.

However, from the approach it is clear that this modified formulation cannot take care of cases where $\tau<0$. This can be handled by using the symmetry property of the ambiguity function and defining a modified distribution function

$$
\begin{array}{rlrl}
\operatorname{Pmod}_{\operatorname{mov}}(\tau, v) & =p(\tau, v)+p(-\tau,-v) & & \text { for } \tau>0 \\
& =0 & \text { elsewhere }
\end{array}
$$

\section{RESULTS}

The formulation of the previous section provides us with a tool for designing signals of specified families, the compact FSK bursts in this case, for optimal rejection of arbitrary 2-D clutter distributions. In this section the usefulness of the formulation is demonstrated by application against assumed representative clutter distributions. The effectiveness can easily be judged by examining the ambiguity behaviour.

We present some representative results in Fig. 1-4 which show the ambiguity function for the optimum FSK bursts for different burst lengths and clutter distributions. For ease in visualization the plots show only the region of interest. The absence of any significant sidelobes in the region of the delay-Doppler plane occupied by the clutter is quite evident and clearly demonstrate the effectiveness of the method. 
The clutter distribution for the optimum signal, shown in Fig. 1 and 2 , are similar in description in the normalized signal duration frame and the increase in the burst length must lead to a commensurate improvement. This can be easily seen in their ambiguity plots. This is also confirmed by the improvement in SIR, normalized with reference to an uncoded burst, which has a value of 10.6 for the 5-pulse burst and improves to 45.5 for the 13-pulse case.

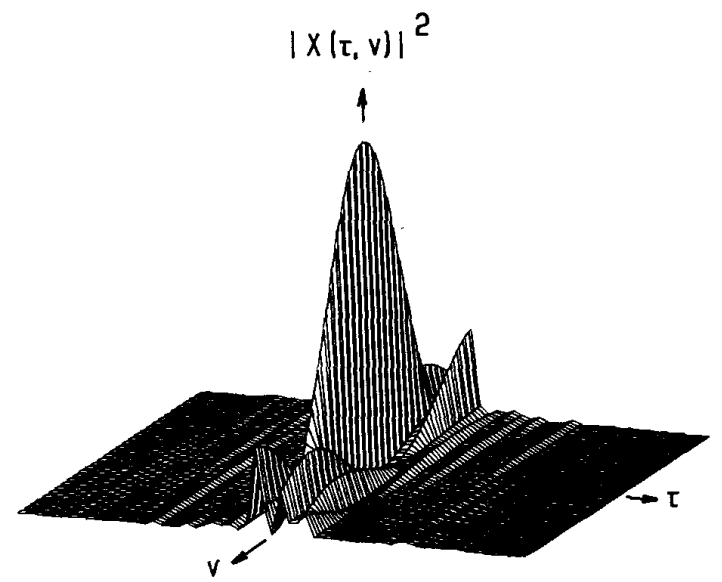

Fig.1 - The ambiguity function of the optimal 5pulse FSK burst, against uniform clutter distributed in a rectangular region given by $1<\tau<5$ and $-1 / N T<v<1 / N T$.

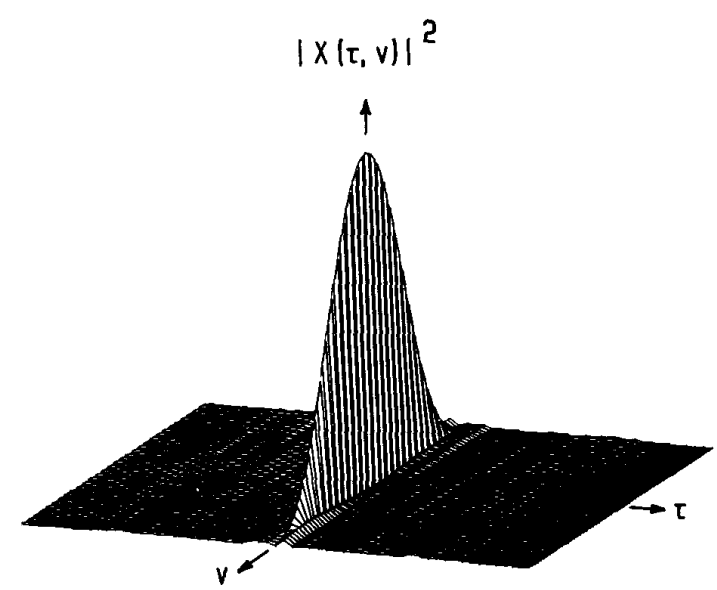

Fig.2 - The ambiguity function of the optimal 13pulse FSK burst, against uniform clutter distributed in a rectangular region given by $1<\tau<13$ and $-1 / N T<v<1 / N T$.
Fig. 3 and 4 show the ambiguity function of the optimum 13-pulse burst against different clutter distributions as mentioned. The distribution have been so chosen so as to cover different delay-Doppler clutter occupations, and thus indicates the effectiveness of the signal format and the optimization algorithm to adapt to any general clutter distribution. The workability of the method can be easily judged by the ambiguity behavior obtained.

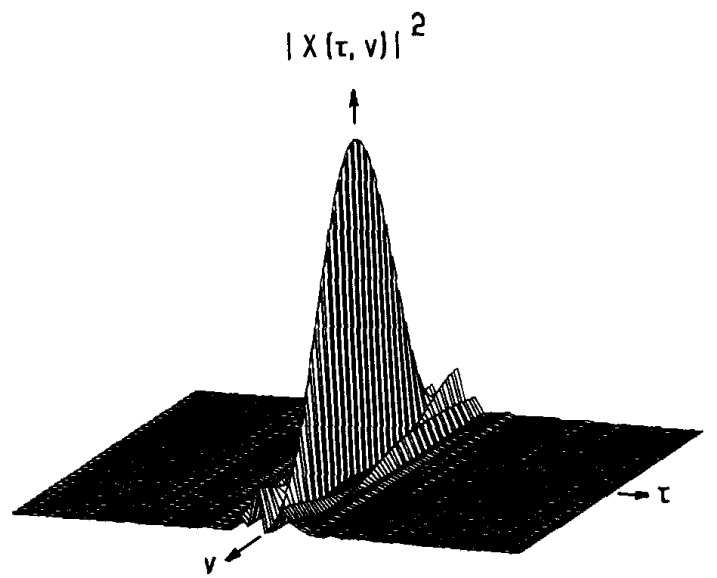

Fig.3 - The ambiguity function of the optimal 13pulse FSK burst, against uniform clutter distributed in a rectangular region given by $1<\tau<5$ and $-1 / N T<v<1 / N T$.

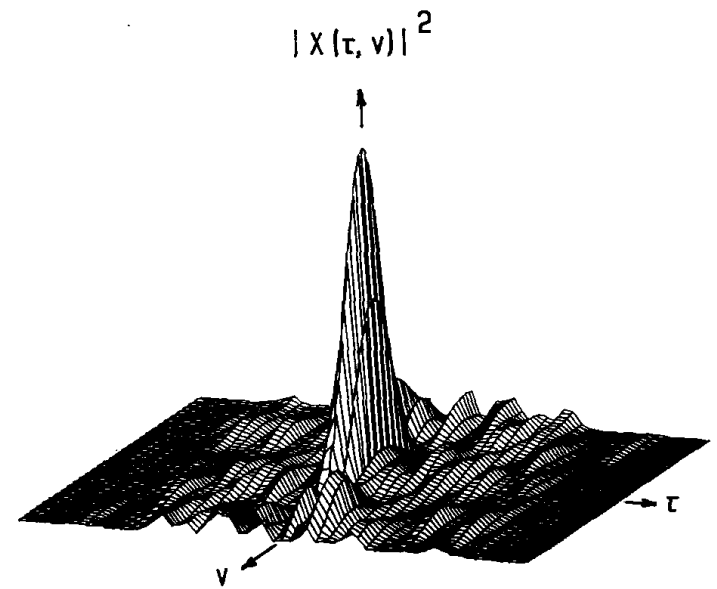

Fig.4 - The ambiguity function of the optimal 13pulse FSK burst, against uniform clutter distributed in a rectangular region given by $0<\tau<1$ and $-2 / \mathrm{NT}<v<2 / \mathrm{NT}$. 


\section{CONCLUSIONS}

In summary, the problem of optimum clutter rejection in a given region of the delay-Doppler plane has been solved. The approach is based on optimizing frequency-coded pulse train transmitted signals, keeping the receiver filter matched to this signal all the time. This reduces the dimensionality of the problem leading to large saving in computational efforts. This factor together with the simplification achieved usirg the piecewise constant property of the signal holds the potential for adaptive signal optimization in time-varying clutter situations.

The actual design of the signal depends to a large extent on the numerical optimization algorithm. Use of a faster converging algorithm will be an added advantage and will contribute much to the application in time varying clutter environment. However, the types of functions encountered here are highly non-linear and have a large no. of local minima. The design of an efficient optimization algorithm, for such functions, is quite difficult and the authors of this paper are currently working towards developing more efficient algorithms for the class of functions encountered in this types of signal design problems.

\section{REFERENCES}

[1] A.W. Rihaczek, Principles of High-Resolution Radar. New York: McGraw Hill, 1969.

[2] H. Urkowitz, "Filters for detection of small Radar signals in Clutter", Journal Appl. Phys., Vol. 24 (Aug. 1953), pp. 1024-1031.

[3] G.L. Turin, "An Introduction to Matched filters", IRE Trans. Information Theory, IT 6 (June 1960), pp. 311-329.

[4] R. Manasse, "The use of Pulse Coding to Discriminate against clutter", MIT Lincoln Lab., Group Rept. 312-12(Rev.1), June 1961.

[5] M. Ares, "Radar Waveforms for Suppression of Extended Clutter", IEEE Trans. Aerospace and Electronic Systems, AES-3 (Jan. 1967), pp. 138-141.
[6] D.F. Delong and E.M. Hofstetter, "On the design of Optimum Radar Waveforms for Clutter Rejection", IEEE Trans. Information Theory, IT-13 (July 1967), pp. 454-463.

[7] D.F. Delong and E.M. Hofstetter, "The design of Clutter-Resistant Radar Waveforms with limited Dynamic Range", IEEE Trans. Information Theory, IT-15 (May 1969), PP. 376-385.

[8] C.A. Stutt and L.J. Spafford, "A 'best' Mismatched Filter Response for Radar Clutter Discrimination", IEEE Trans. Information Theory, IT-14 (Mar. 1968), pp. 280-287.

[9] L.J. Spafford, "Optimum Radar Signal Processing in Clutter", IEEE Trans. Information Theory, IT-14 (Sep. 1968), pp. 734-743.

[10] L.J. Ziomek and L.H. Sibul, "Maximization of Signal to Interference Ratio for a Doublyspread target: Problems in nonlinear Programming", Signal Processing, 5 (July 1983), pp. 355-368.

[11] C.E. Cook and M. Bernfeld, Radar Signals: An Introduction to Theory and Application. New York: Academic Press, 1967.

[12] R.M. Mersereau and T.S. Seay, "Multiple Access Frequency Hopping Patterns with Low Ambiguity", IEEE Trans. Aerospace and Electronic Systems, AES-17 (July 1981), pP. $571-578$

[13] E.L. Titlebaum, "Time-Frequency Hop Signals Part I: Coding based upon the Theory of Linear Congruences", IEEE Trans. Aerospace and Electronic Systems, AES-17 (July 1981), pp. 490-493.

[14] E.L. Titlebaum and L.H. Sibul, "TimeFrequency Hop Signals Part II: Coding based upon Quadratic Congruences", IEEE Trans. Aerospace and Electronic Systems, AES-17 (July 1981), pp. 494-500.

[15] C.N. Oligboh and M.H. Ackroyd, "Linear frequency coded sequences", IEE Proc. PartG, Vol. 127 (Aug. 1980), pp. 191-198. 